\title{
Specificity of using wireless technologies in Earth remote sensing
}

\author{
Michail Kazakov ${ }^{1}$, Fedor Kazakov ${ }^{1}$, and Natalia Sirotinina ${ }^{1}$ \\ ${ }^{1}$ Siberian Federal University, Computer Science Department, 79 Svobodny pr., 660041 Krasnoyarsk, \\ Russia
}

\begin{abstract}
The article discusses an approach to organizing the interaction of nodes of spacecraft onboard data transmission networks based on wireless communication technologies. The main quality indicators and requirements for traffic control in on-board networks are formulated taking into account the peculiarities of the wireless network nodes interaction. The results of modeling various algorithms of transmission medium access control for one of the common protocols for organizing wireless networks (IEEE 802.15.4) are presented..
\end{abstract}

\section{Introduction}

Systems of remote sensing (ERS) based on spacecraft are one of the priority areas for the development of science and the space industry [1]. The number and density of satellites that survey in various bands of electromagnetic spectrum with a resolution from several kilometers to several tens of centimeters is constantly growing [2-6] .

One of the main trends in the development of ERS in recent years is the use of multi- and hyperspectral optoelectronic systems operating in both visible and infrared ranges. This allows us to observe the earth's surface using hundreds of spectral channels. At the same time, this trend leads to an increase in the information performance required to process and transmit traffic that is generated by remote sensing systems on board a spacecraft. The information performance of modern spacecraft can reach 2 Gbit / s. For comparison, in devices of twenty years ago this parameter rarely exceeded tens of Mbit / s [7].

Currently, research is mainly focused on the information transmission over space-to-Earth communication channels. At the same time, development of spacecraft onboard data transmission systems taking into account the requirements of fault tolerance and speed, is also an important task [10].

One of the most promising approaches in this area is the use of wireless networks. It allows to reduce the use of cable bundles in onboard networks, the mass of which is up to $10 \%$ of the total mass of the spacecraft [8-9].

In contrast to conventional wire technologies, in wireless networks the data transmission medium is shared by all on board nodes of the spacecraft. When determining the effective bit rate, consideration must be given to the sharing of transmission medium. Depending on the wireless technologies used, the bit rate can be tens of Gbps.

\footnotetext{
${ }^{1}$ Corresponding author: mkazakov@sfu-kras.ru
} 
When choosing protocols and developing algorithms for accessing the data transmission medium, it is necessary to take into account the situation when a delay-critical traffic (for example, control signals) and a large data amount from the spacecraft payload (for example, from equipment of hyperspectral optoelectronic systems) must be transmitted simultaneously.

In public information networks, this problem is solved by using various algorithms for traffic prioritization and setting quality of service parameters. At the same time, significant restrictions on the traffic parameters are imposed by the used protocols of the physical data transmission medium access.

IEEE 802.15.4 [11] is one of the standards corresponding to the specific application in the spacecraft on-board network. It specifies the protocol that defines the physical layer and the media access control algorithm. This standard defines two modes of access to the transmission medium, namely concurrent and guaranteed access. The first is based on the carrier sense multiple access with collision avoidance (CSMA / CA), which does not allow control of QoS parameters and allows packet loss. The second mode enables to reserve time intervals for transmitting information from a limited number of nodes. In this case, guaranteed access mode with specified characteristics is provided.

To evaluate the performance of this standard, a model was developed using the OMNeT simulation environment.

It is proposed to use the average delay in packet transmission and the percentage of packet loss due to denial of access to the transmission medium as the evaluation criteria.

An experimental study of the model showed a number of disadvantages that affect the possibility of using this standard in spacecrafts.

The results of experiments with the original IEEE 802.15.4 are shown in Figure 1. As you can see, percentage of packet loss begins to grow starting from the number of nodes equal to seven. By the time fourteen nodes are involved in transmission, the loss percentage becomes more than $12 \%$ and continues to grow.

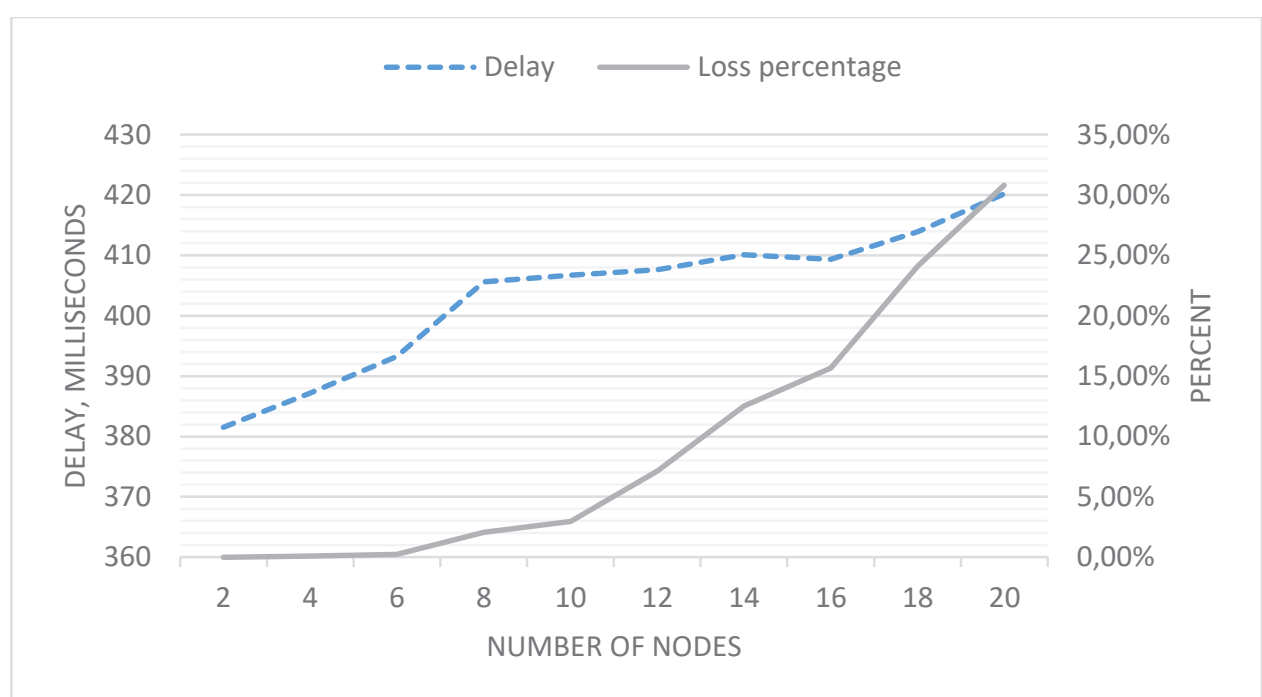

Fig. 1. Latency and percentage of packet loss for concurrent access

In the guaranteed access mode, a zero percentage of packet loss is provided for no more than seven nodes. The rest of the nodes cannot participate in data transmission in the guaranteed access mode.

To avoid packet loss when using the guaranteed access mode, a number of modifications of the guaranteed access slot allocation algorithm have been proposed. The modifications consist 
in the distribution of guaranteed slots using various algorithms. Algorithms of random selection, ring shift, priority algorithm and class prioritization were selected as the most promising.

The proposed solution made it possible to avoid the loss of packets for any number of nodes participating in the transmission. However, this increased delays in packet transmission. As you can see in fig. 2, the delay depends on the number of nodes and the algorithm used.

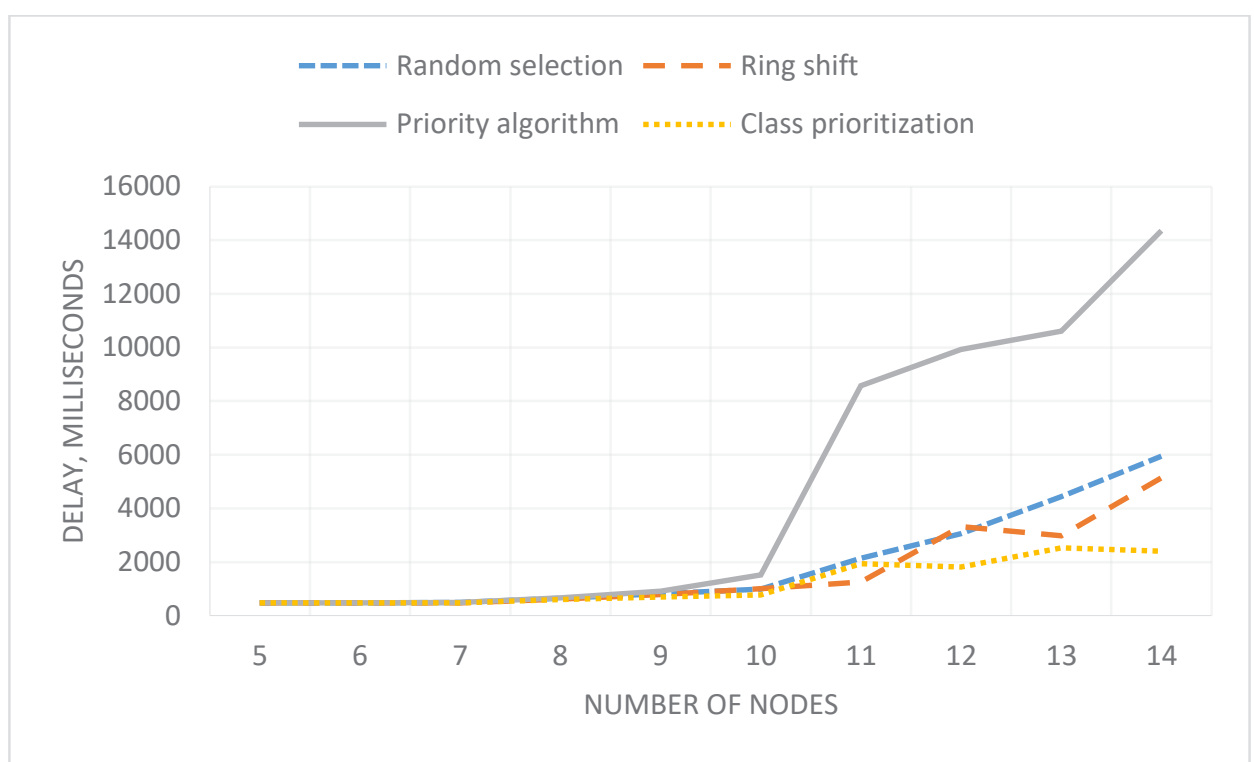

Fig. 2. Delays for different access algorithms

The random selection method is characterized by a graph consisting of two linearly increasing sections. The section corresponding to ten or more nodes participating in the transmission is characterized by faster growth.

For the circular shift method, the delay depends on the queue shift parameter. In general, the pattern of delay growth is similar with the random selection method. Some uneven growth is observed due to effects related to the multiplicity of the shift length to the total number of nodes.

The main way to reduce latency growth is to use a priority algorithm. In this case, different types are distinguished in the total traffic depending on the consumer category. A reasonable priority is assigned to each traffic type. Experiments have shown a significant increase in the average delay for this algorithm when the number of nodes increases over 10 (Figure 3). 


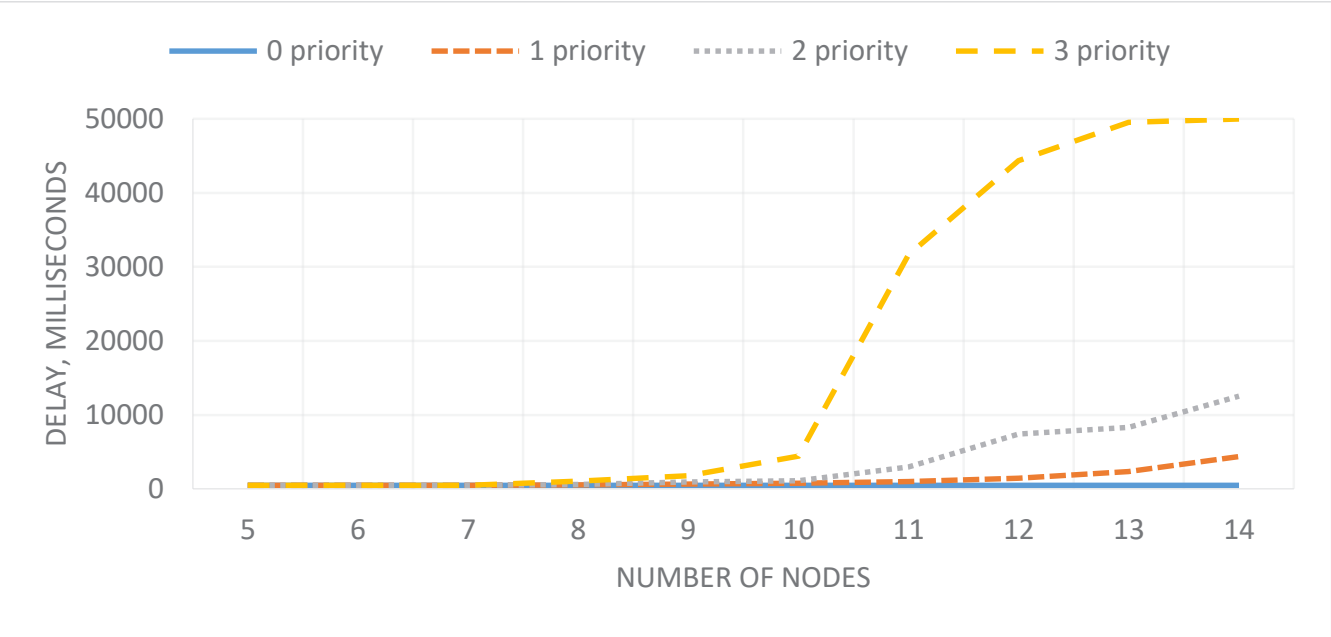

Fig. 3. Dependence of delay for different priorities on the number of nodes in the case of using the priority algorithm

This is due to a latency increase for low priority nodes. However, for the nodes with the highest (zero) priority, the delay time increases insignificantly (Figure 3). For nodes of the first and second priorities, the delay also increases, starting from the tenth node.

\section{Conclusion}

The article analyzes the operation of wireless sensor networks based on the IEEE 802.15.4 standard in a situation where high data transmission reliability is required and network overload is observed. The goal of the analysis is to determine the effect of number of nodes on the percentage of packet loss and latency, and to suggest approaches for improving network performance.

Simulation of the proposed modifications to distribution control of guaranteed time slots showed a complete absence of packet loss, which confirms the high reliability of data transmission even with a large number of nodes.

A side effect of this is an increase in packet transmission delay (latency). This can be partially compensated for by using prioritized algorithms and optimizing node priorities.

The obtained results allow us to draw a conclusion about the applicability of the proposed modified protocol when building on-board wireless networks of spacecraft, including for solving remote sensing tasks.

\section{References}

1. I.N. Gansvind, Small satellites in remote sensing of the Earth, Exploration of the Earth from Space, 5, pp. 82-88, (2019)

2. A.V. Shpak, O.A. Zheleznyak, Geoinformation analysis of remote sensing data to forecast the possibility of forest fires, Astronomical School's Report, 7, (2011)

3. H. LIANG, C. ZHANG, Z. MENG, Real-time observation monitoring and analysis network, ANNEX II, 2, (2008)

4. R. Dicati, Earth Remote Sensing, Stamping the Earth from Space, pp.293-359, (2017) 
5. Y.A. Maglinets, G.M. Tsibul'skii, M.V. Noskov, Current Trends of Development of the Regional Systems of Earth Remote Sensing, Sib. Fed. Univ. Eng. technol., 9(7), 10121018. (2016)

6. N.E. Zimmermann, R.A. Washington-Allen, R.D. Ramsey, M.Schaepman, Modern Remote Sensing for Environmental Monitoring of Landscape States and Trajectories, A Changing World, pp. 65-91, (2007)

7. G. N. Maltsev, I. A. Kozinov, Transfer of Hyperspectral Video Data of Earth Remote Sensing via Radio Channels of Limited Capacity, INFORMATION CONTROL SYSTEMS, 2, pp 74-83, (2019).

8. R. A Magness, Comparison of can and bluetooth protocols a study for application of can over bluetooth for wireless on-board data handling for a spacecraft sensor network, DASIA of ESA Special Publication, (2003)

9. M. Meltzer, The Cassini-Huygens visit to Saturn: An HistoricMission to the Ringed Planet, Springer, (2015)

10. D.S. Andrahitov, S.A. Gezha, A.A. Filimonov, PROBLEM ISSUES OF INCREASING THE ACCURACY OF MEASUREMENTS ONBOARD SPACE VEHICLES, Modern High Technologies, 19, (2020)

11. S. Li, Comparative Analysis of Infrastructure and Ad-Hoc Wireless Networks, ITM Web of Conferences, (2019) 